\title{
SOP Proximale Femurfraktur
}

Michael J. Raschke, Jens Everding

\section{Einleitung}

Frakturen des proximalen Femurs umfassen die Femurkopffrakturen, Schenkelhalsfrakturen und die Frakturen der trochantären sowie subtrochantären Region. In Deutschland zeigt sich eine altersabhängige, exponentiell ansteigende Inzidenz dieser Frakturen von $0,06 \%$ bei den 60 - bis 64 -Jährigen, bis hin zu 1,32\% im Alter von 85 Jahren oder höher [7].

Insgesamt handelt es sich bei den proximalen Femurfrakturen um die häufigste Ursache eines stationären Krankenhausaufenthalts von Patienten im Alter über 64 Jahre, bedingt durch die resultierende Immobilität mit notwendiger stationärer und operativer Versorgung [12]. Aufgrund des demografischen Wandels ist in Zukunft von einer deutlichen Zunahme dieser Verletzungsarten auszugehen [4]. Daher ist das Wissen um die SOPs bei proximalen Femurfrakturen im klinischen Alltag von großer Bedeutung.

\section{Cave}

Die 90-Tages-Mortalität bei proximalen Femurfrakturen beträgt 7,9\% [6].

\section{Femurkopffraktur (Pipkin-Fraktur)}

Femurkopffrakturen sind seltene Verletzungen, die häufig auf ein Hochenergietrauma zurückzuführen und mit Hüftkopfluxationen oder Azetabulumfrakturen assoziiert sind. Insgesamt wird in 5-15\% der traumatischen Hüftkopfluxationen eine Femurkopffraktur beobachtet [3]. Häufigster Unfallmechanismus ist der Verkehrsunfall mit resultierender „dashboard injury“, betroffen sind vor allem junge Menschen, wobei das männliche Geschlecht überwiegt. Weitere Unfallmechanismen, die zu dieser Verletzung führen, sind Verkehrsunfälle mit Fußgängerbeteiligung, Sportunfälle und Stürze aus großer Höhe.

\section{Schenkelhalsfraktur}

Die Schenkelhalsfraktur ist eine typische Verletzung des alten Menschen. Ursache ist in der Mehrzahl der Fälle ein Bagatelltrauma mit Sturz aus geringer Höhe, wobei die Inzidenz dieser Verletzung bei den über 85-jährigen Frauen deutlich höher liegt als in der altersgleichen männlichen Gruppe (2543 vs. 1370) [5]. Prädisponierend ist die Osteoporose.
Deutlich seltener ist eine Schenkelhalsfraktur Folge eines Hochenergietraumas, welches vor allem junge männliche Patienten betrifft. Klinisch imponiert die Schenkelhalsfraktur durch Schmerzen in der Leistenregion und die Unfähigkeit, das betroffene Bein von der Untersuchungsliege anzuheben. Die aktive Beweglichkeit im Hüftgelenk ist aufgehoben. In den meisten Fällen ist aufgrund des Muskelzugs eine Fehlstellung mit Beinverkürzung und Außenrotation nachzuweisen.

Die Schenkelhalsfraktur ist die bedeutendste alterstraumatologische Verletzung und geht einher mit einem hohen Risiko von perioperativer Morbidität und Mortalität [11].

\section{Fraktur der peri- und subtrochantären Region}

Die Hälfte der proximalen Femurfrakturen sind in der peri- bzw. subtrochantären Region lokalisiert. Hinsichtlich der Patientencharakteristika, des Unfallmechanismus und der Klinik besteht eine Analogie zu den Schenkelhalsfrakturen, wenngleich sich die Strategie der operativen Behandlung deutlich unterscheidet.

\section{Präoperative Diagnostik und Klassifikation}

- Erhebung der Unfallanamnese

- subjektive Beschwerden

- körperliche Untersuchung:

- Fehlstellung des Beines: dorsale Hüftkopfluxation mit Hüftkopffraktur: Flexion, Adduktion, Innenrotation; Schenkelhalsfraktur und peri- bzw. subtrochantäre Frakturen: Beinverkürzung und Außenrotation

- Bewegungseinschränkung (Cave: Die detaillierte Bewegungsprüfung sollte aufgrund der Schmerzen unterbleiben.)

- Überprüfung der körperfernen Durchblutung, Motorik und Sensibilität

- bildgebende Diagnostik:

- tiefe Beckenübersichtsaufnahme mit Messkugel und Hüfte axial nach Sven Johansson

- bei Femurkopffraktur: CT-Diagnostik zur Abschätzung des Verletzungsausmaßes (Größe des Fragments, freie Gelenkkörper) 
- bei peri- bzw. subtrochantären Frakturen: Bildgebung des gesamten Femurs einschließlich Knie (Einbringen zephalomedullärer Implantate)

- Komplettierung der (Medikamenten-)Anamnese. Betroffen sind häufig alte Patienten mit Antikoagulation!

- weitere Diagnostik entsprechend Unfallmechanismus und Untersuchungsbefund

Cave

Verletzung des Knies bei Femurkopffrakturen („dashboard injury“).

\section{Femurkopffraktur}

Die international am häufigsten angewandte Klassifikation der Hüftkopffrakturen erfolgt nach Pipkin ( $\mathbf{A} \mathbf{A b} \mathbf{b} . \mathbf{1}$ ) unter Berücksichtigung der Lagebeziehung der Fraktur zur Belastungszone des Femurkopfs.

Neben einem Beitrag zur Therapieentscheidung besitzt die Einteilung nach Pipkin ebenso eine prognostische Bedeutung [14]:

- Pipkin Typ I: Fraktur kaudal der Fovea capitis

- Pipkin Typ II: Fraktur kranial der Fovea capitis

- Pipkin Typ III: Pipkin Typ I/II in Kombination mit Schenkelhalsfraktur

- Pipkin Typ IV: Pipkin Typ I/II in Kombination mit Azetabulumfraktur

Die AO-Klassifikation sowie die weniger gebräuchliche Brumback-Klassifikation sind weitere Möglichkeiten zur Einteilung der Hüftkopffrakturen. Letztere umfasst eine genauere Beschreibung der Frakturmorphologie einschließlich der potenziellen Luxationsrichtung des Kopfes.

\section{Schenkelhalsfraktur}

Pauwels entwickelte 1935 eine mechanisch begründete Klassifikation der Schenkelhalsfrakturen, basierend auf den vorliegenden Scherkräften. Insgesamt weist diese Klassifikation eine hohe Interobservervariabiliät auf, bei fehlender klinischer Relevanz [9].

Der Pauwels-Winkel ergibt sich hierbei als Differenz aus Horizontale und Scherwinkel:

- Pauwels Typ I: $<30^{\circ}$

- Pauwels Typ II: 30-50

- Pauwels Typ III: >50

Garden beschrieb 1961 die Schenkhalsfrakturen in Abhängigkeit vom Dislokationsgrad bzw. der zentralen Trabekelausrichtung im a.-p. Röntgenbild in vier Graden. Die Interobservervariabilität ist hoch, lässt sich allerdings durch Unterteilung in nicht dislozierte (Typ I/II) und dislozierte (Typ III/IV) Fraktur verringern. Die klinische Bedeutung dieser Subklassifikation liegt in der höheren Hüftkopfnekroserate bei dislozierten Frakturen.
Im klinischen Alltag ist die Garden-Klassifikation wichtiger Bestandteil für die therapeutische Entscheidungsfindung (> Abb. 2):

- Garden Typ I: valgisierende und eingestauchte Fraktur, der Winkel zwischen Kopftrabekel und medialer Kortikalis beträgt $>160^{\circ}$

- Garden Typ II: nicht dislozierte Fraktur in der a.-p. Ebene

- Garden Typ III: varisch und leicht dislozierte Fraktur, medial stehen die Trabekel noch in Kontakt

- Garden Typ IV: vollständig dislozierte Fraktur, kein Trabekelkontakt

Eine eher akademische Bedeutung kommt der AO-Klassifikation (1990) der Schenkelhalsfrakturen zu, die den Dislokationsgrad und die Lokalisation berücksichtigt.

\section{Fraktur der peri- und subtrochantären Region}

Pertrochantäre Femurfrakturen werden anhand der AOKlassifikation eingeteilt:

- Typ 31 A1: stabiler Frakturtyp, die mediale Kortikalis ist einfach frakturiert, keine Beteiligung des Trochanter minor

- Typ 31 A2: instabiler Frakturtyp, die mediale Kortikalis ist mehrfach frakturiert, Mitbeteiligung des Trochanter minor, Trümmerzone zwischen Trochanter major und Trochanter minor
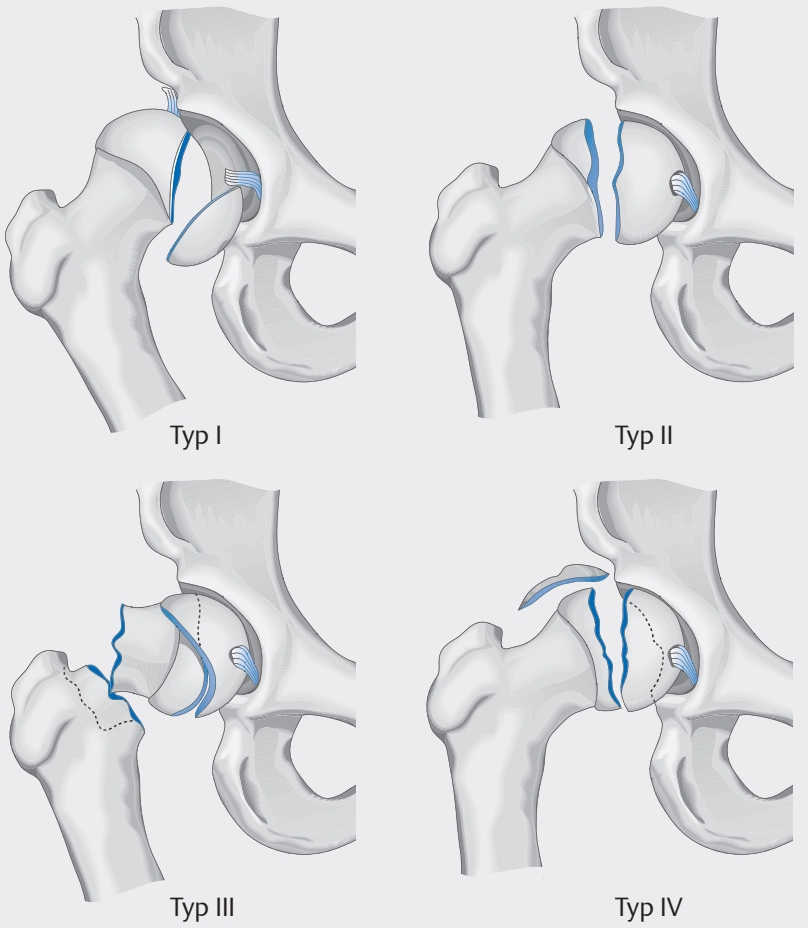

- Abb. 1 Femurkopffrakturen. Klassifikation nach Pipkin. (Wirth C), Mutschler W. Praxis der Orthopädie und Unfallchirurgie. Stuttgart: Thieme; 2007) 


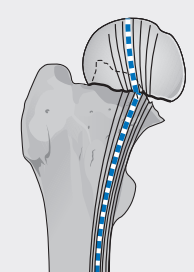

Typ I

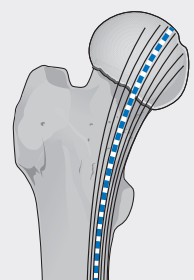

Typ II

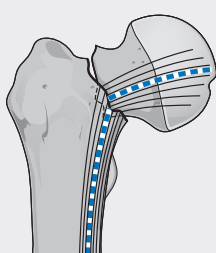

Typ III

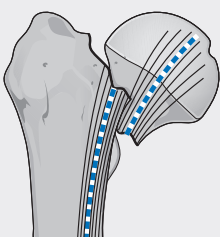

Typ IV
- Abb. 2 Mediale Schenkelhalsfrakturen. Klassifikation nach Garden. (Quelle: Ruchholtz S, Wirtz DC. Orthopädie und Unfallchirurgie essentials. Stuttgart: Thieme; 2012)

- Typ 31 A3: instabiler Frakturtyp, die mediale und laterale Kortikalis ist frakturiert, inverse oder oblique Fraktur

\section{Indikationsstellung und Verfahrenswahl}

\section{Femurkopffraktur}

Ziel ist die Wiederherstellung einer stabilen Hüftgelenksituation mit anatomischer Gelenkkongruenz sowie ggf. auch Entfernung von interponierenden Fragmenten aus dem Azetabulum. Nichtdislozierte Pipkin-Typ-I-Frakturen, die sich geschlossen anatomisch reponieren lassen und im Anschluss stabil sind sowie keine interponierenden Strukturen aufweisen, können konservativ behandelt werden.

In den meisten Fällen ist jedoch eine operative Versorgung erforderlich, die eine Fragmentrefixation mit 2,0bis 3,5-mm-Kleinfragmentschrauben bzw. Knochenfixierstiften umfasst. In Abhängigkeit von der Größe des Frag- ments, der Anzahl der Frakturfragmente und der Lokalisation des Fragments in Bezug auf die gelenktragende Region ist alternativ eine Fragmentresektion möglich. Impressionsfrakturen müssen ggfs. hochgestößelt und mit Spongiosa unterfüttert werden. Bei älteren Patienten empfiehlt sich anstelle einer kopferhaltenden Operation die endoprothetische Versorgung.

Bei Pipkin-Typ-III-Frakturen sollte bei jungen Patienten zunächst die Verschraubung der Schenkelhalsfraktur mittels kanülierten Großfragment-Spongiosaschrauben (7,3 mm oder 7,0 mm) erfolgen, bevor über einen vorzugsweise anterioren oder anterolateralen (WatsonJones-)Zugang eine kopferhaltende Versorgung der Kopffraktur durchgeführt wird.

Für die Versorgung von Pipkin-Typ-IV-Frakturen bietet sich insbesondere bei Beteiligung des hinteren Pfannenrands der Kocher-Langenbeck-Zugang an, mit ggf. auch chirurgischer Hüftgelenkluxation und Trochanter-FlipOsteotomie zur Adressierung des Kopffragments. Das genaue Vorgehen einschließlich bevorzugter Zugangswahl beschreibt $>$ Tab. 1 .

Cave

Die mit der Hüftkopffraktur assoziierte Luxation des Femurkopfs ist ein absoluter Notfall und bedarf der sofortigen geschlossenen bzw. offenen Reposition.

\section{Schenkelhalsfraktur}

Eine konservative Therapie von Schenkelhalsfrakturen ist nur in Ausnahmefällen indiziert. Valgusimpaktierte Schenkelhalsfrakturen können unter suffizienter Schmerztherapie und engmaschigen Röntgenverlaufskontrollen zum Ausschluss einer sekundären Dislokation unter Vollbelastung nichtoperativ behandelt werden. Aufgrund der hohen sekundären Dislokationsrate mit bis zu 56\% [16] wird im eigenen Vorgehen jedoch die pro-

> Tab. 1 Therapiealgorithmus bei Femurkopffrakturen.

\begin{tabular}{|c|c|c|}
\hline Pipkin & operatives Vorgehen & Zugang \\
\hline \multirow[t]{2}{*}{ I } & 1. Fragmentrefixation oder Fragmentresektion* & anterior \\
\hline & 2. Endoprothese beim älteren Patienten & anterior, anterolateral, lateral, posterior \\
\hline \multirow[t]{2}{*}{ II } & 1. Kopferhaltende Osteosynthese oder Fragmentresektion* & anterior \\
\hline & 2. Endoprothese beim alten Patienten & anterior, anterolateral, lateral, posterior \\
\hline \multirow[t]{2}{*}{ III } & $\begin{array}{l}\text { 1. Schenkelhalsosteosynthese, folgend Fragmentrefixation } \\
\text { oder Fragmentresektion* }\end{array}$ & anterior-lateral \\
\hline & 2. Endoprothese beim alten Patienten & anterior, anterolateral, lateral, posterior \\
\hline \multirow[t]{2}{*}{ IV } & $\begin{array}{l}\text { 1. Refixation Pfannenfragment, folgend Fragmentrefixation } \\
\text { oder Fragmentresektion* }\end{array}$ & posterior \\
\hline & 2. Endoprothese beim alten Patienten & posterior, chirurgische Hüftluxation, Flip-Osteotomie \\
\hline
\end{tabular}


phylaktische (perkutane) Schraubenosteosynthese favorisiert.

Grundsätzlich sollte das Ziel der operativen Versorgung von Frakturen des Schenkelhalses das frühzeitige Wiedererlangen der Mobilität des Patienten sein, um Komplikationen und Folgeerkrankungen (Thrombose, Pneumonie, Dekubitus) zu vermeiden. Eine frühzeitige, notfallmäßige operative Versorgung ist laut Lehrmeinung von großer Bedeutung. In verschiedenen Studien konnte gezeigt werden, dass ein erhöhtes Risiko von perioperativen Komplikationen bei verzögerter operativer Therapie vorliegt.

Insbesondere bei kopferhaltender Osteosynthese besteht eine erhöhte Gefahr der sekundären Hüftkopfnekrose, wenngleich hierzu eine gute wissenschaftliche Evidenz nicht vorliegt [8]. Ebenso reduziert sich bei einer frühzeitigen endoprothetischen Frakturversorgung die postoperative Komplikationsrate sowie Sterblichkeit der Patienten [12]. Umstritten ist die Druckentlastung des Kapselhämatoms bei geschlossener Reposition und Osteosynthese [2].

Der Therapiealgorithmus bei Schenkelhalsfrakturen wird in $\mathbf{A b b} \mathbf{3}$ dargestellt.

\section{Fraktur der peri- und subtrochantären Region}

Für die operative Versorgung von peri- und subtrochantären Frakturen stehen extramedulläre und zephalomedulläre Implantate zur Verfügung. Die Wahl des Implantats basiert auf der Frakturmorphologie.

Stabile peritrochantäre Frakturen mit medialer Abstützung (AO-31.A1-Frakturen) können mit extramedullären Implantaten versorgt werden. Implantat der Wahl ist die DHS (dynamische Hüftschraube) bzw. DHK (dynamische Hüftklinge), welche beide eine mediolaterale Dynamik und bei korrekter „Center-Center-“Platzierung eine belastungsstabile Nachbehandlung erlauben.

Frakturen mit Beteiligung beider Trochanteren müssen als instabil betrachtet werden (AO 21.A2) und weisen neben einer mediolateralen auch eine kraniokaudale sowie in Abhängigkeit von der intertrochantären Trümmerzone eine Rotationsinstabilität auf. In diesem Falle ist die Versorgung mittels zephalomedullärem Implantat (GammaNagel, PFNa, TFN) empfehlenswert. Alternativ ist die Verwendung einer DHS bzw. DHK mit Trochanterstabilisierungsplatte möglich. Biomechanisch bieten die zephalomedullären Implantate aufgrund der höheren Stabilität infolge der verminderten Distanz zwischen Hüftkopf und Implantat mit resultierendem geringerem Hebelarm einen Vorteil; klinisch konnte dies bislang nicht nachgewiesen werden [10].

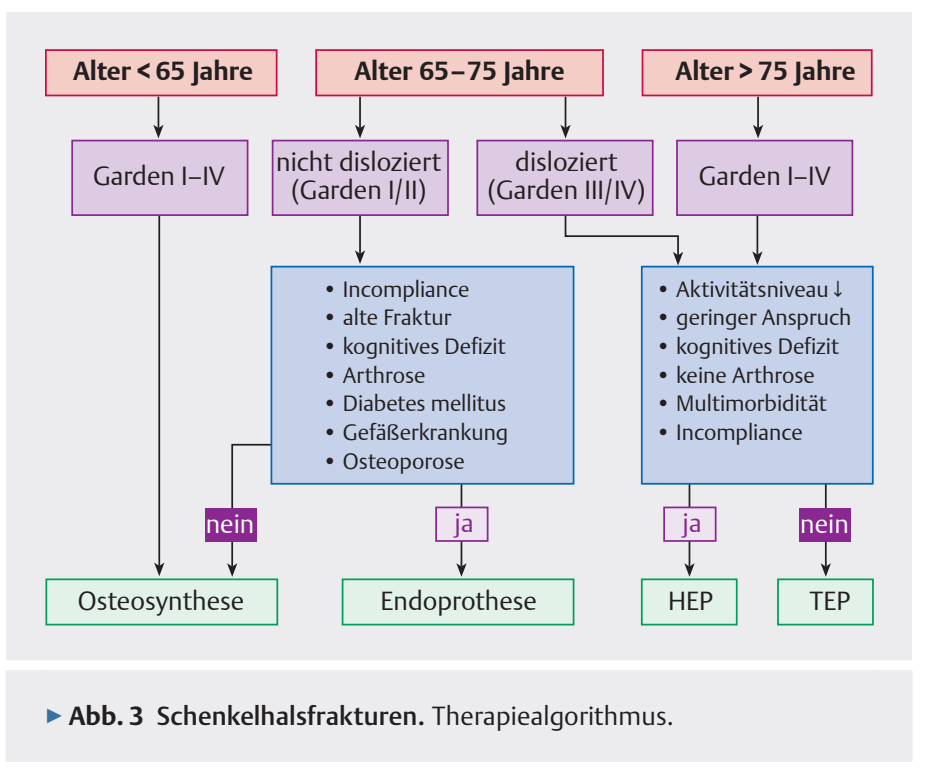

Frakturen mit inversem bzw. obliquem Frakturverlauf (AO 31.A3) sowie Frakturen der subtrochantären Region sollten mittels langem zephalomedullärem Nagel versorgt werden. Häufig liegt aufgrund des Muskelzugs eine grobe Dislokation der Frakturfragmente vor, sodass regelhaft ein offenes Vorgehen empfehlenswert ist. Hierbei erfolgen zunächst die Reposition und Fixierung der Fragmente mittels Draht- oder Kabelcerclage, bevor der Marknagel eingebracht wird. In Abhängigkeit von der Knochenqualität ist die additive Zementaugmentation der extramedullären bzw. zephalomedullären Implantate empfehlenswert.

Eine primäre endoprothetische Versorgung sollte bei peri- und subtrochantären Frakturen - insbesondere bei Verlust der medialen Abstützung - vermieden werden. Neben dem erhöhten Risiko einer Varusimplantation und eines Rotationsfehlers ist die Offset-Rekonstruktion deutlich erschwert [1]. Insgesamt zeigt sich bei Patienten mit endoprothetischer Versorgung nach Frakturen der periund subtrochantären Region eine gesteigerte Komplikationsrate [13].

\section{Aufklärung und Komplikationen}

\section{Allgemeine Operationsrisiken}

Allergie, Thrombose, Lungenembolie, Fettembolie, Verletzung von benachbarten Strukturen (Gefäße/Nerven/ Sehnen/Muskeln), Blutung, Nachblutung, Infektion, Wundheilungsstörung, Lagerungsschäden, Folgeoperation, Immobilität, Schmerzen. 


\section{Spezielle Operationsrisiken}

- sekundärer Repositionsverlust

- Hüftkopfnekrose (bei konservativer Therapie bzw. Osteosynthese)

- Cut-out/Cut-through (bei extra- und intramedullären Implantaten)

- Läsion des N. ischiadicus (bei Hüftkopffraktur bzw. dorsalem Zugang)

- intraoperative/periimplantäre/periprothetische Fraktur

- Arthrose

- Pseudarthrose

- Trendelenburg-Hinken

- Beinlängendifferenz

- Rotationsfehler des Beines (bei Prothesen- und Marknagelimplantation)

- Prothesenluxation

- Prothesenverschleiß (Abrieb)

- Fehllage der Prothesenpfanne (Inklination/Anteversion)

- Fehlimplantation Prothesenschaft (Varus-/Valgusimplantation, Rotationsfehler, Offset-Rekonstruktion)

- Implantatlockerung, Implantatversagen, Implantatbruch

- Notwendigkeit des Implantatwechsels (Prothesenwechsel)

- Girdlestone-Situation

- Arthrodese

\section{Präoperative Checkliste}

- präoperative (Größen-)Planung der Implantate anhand der Röntgenbilder (Messkugel verwenden!)

- Überprüfung des Vorhandenseins der (Prothesen-)Implantate (einschließlich der nächstkleineren bzw. nächstgrößeren Implantate)

- Kontrolle der Laborbefunde, insbesondere des Gerinnungsstatus

- Bestimmung der Blutgruppe und Bereitstellen von zwei Erythrozytenkonzentraten (bei Prothesenimplantation)

- Markierung der verletzten Seite

\section{Perioperative Maßnahmen}

- perioperative Antibiose (z. B. Cefuroxim 1,5 g i.v. in der Einleitung und intraoperativ alle zwei Stunden)

- Lagerung:

- Hüftkopffraktur: abhängig vom Zugangsweg (anterior, lateral, posterior) in Rückenlage oder Seitenlage mit entsprechender Verwendung von Stützen (Rückenlage) oder Vakuummatratze und Stützen (Seitenlage)
- Schenkelhalsfraktur:

- abhängig vom Operationsverfahren (Osteosynthese, Prothese) und Zugangsweg (anterior, anterolateral, lateral, posterior)

- bei osteosynthetischer Versorgung ggf. Verwendung des Extensionstisches

- bei prothetischer Versorgung Rücken- (anterolateraler Zugang) oder Seitenlagerung (lateraler oder posteriorer Zugang) mit entsprechenden Hilfsmitteln (s. o.) bzw. Extensionstisch bei anteriorem Zugang

- peri-/subtrochantäre Fraktur: Rückenlage auf dem Extensionstisch, das gesunde Bein in Beinschale abduziert auslagern, Oberkörper zur gesunden Seite abduzieren

- Auslagerung der Arme

- Rasur des Operationsgebiets

- Sicherstellung der Durchleuchtungsmöglichkeiten (Hüfte a.-p. und ggfs. Hüfte axial)

- ggf. geschlossene Reposition unter entsprechenden Repositionsmanövern

- steriles Abwaschen des betroffenen Beines, einschließlich der Region auf der verletzten Seite bis zum Bauchnabel

- Abdecken des Beines mittels Vertikalfolie (Extensionstisch) bzw. des beweglichen Beines unter Verwendung von Stockinette und Schlitztuch (Rückenlage bzw. Seitenlage)

- Sauger, Saugersack, monopolarer Elektrokauter, JetLavage (bei prothetischen Eingriffen), je nach zu erwartenden Blutverlust und OP-Dauer ggf. Cell Saver

- Instrumentarium:

- Grundsieb für Knochen- und Weichteileingriff

- Schraubenosteosynthese: Kirschner-Drähte, kanülierte Schrauben (Größe 7,0-/7,3-mm-Spongiosaschrauben)

- extramedulläre/zephalomedulläre Implantate: Kirschner-Drähte, Messlehre, Zielgerät/Zielbügel, Zentrierhülse, Dreistufenbohrer, bzw. Stufenbohrer, Spiralbohrer, Einschlaginstrument/Einschlagbolzen, Führungsstange und Kombihammer, Abstütz-/Kompressionsmutter/Gewebeschutzhülse/ Trokar, Schraubenzieher, Bohrbüchsen, Drehmoment

- Hüft-TEP: TEP-Grundsieb mit Hohmann-Hebel/-Haken, oszillierende Säge, implantatspezifisches Sieb mit Reamer, Einschläger, Raspeln, Probeimplantate (Pfannen, Inlays, Schaft, Kopf) - zementiert/zementfrei

- Team-Time-out nach WHO-Richtlinien

- Redon-Drainagen

- elastische Wickelung des Beines und Anlage eines Spica-Verbands 


\section{Postoperative Maßnahmen und Nachsorge}

Das postoperative Vorgehen nach proximalen Femurfrakturen umfasst folgende Punkte:

- physikalische (AV-Pumpe) und medikamentöse Thromboseprophylaxe ab sechs Stunden postoperativ

- adäquate Schmerztherapie

- Entfernung der Drainagen nach 24-48 Stunden

- Röntgen der Hüfte in zwei Ebenen am zweiten postoperativen Tag

- regelmäßige Wundkontrollen und Verbandswechsel

- physiotherapeutische Beübung (inklusive CPM), manuelle Lymphdrainage:

- Hüftkopffraktur: frühfunktionelle Mobilisierung, Entlastung für sechs bis acht Wochen, ggf. zwölf Wochen bei Versorgung von Impressionsfrakturen mit Unterfütterung, zur Luxationsprophylaxe Limitierung der Hüftflexion bis $60^{\circ}$ sowie Vermeidung von Adduktion über die Mittellinie und forcierter (Innen-)Rotation für sechs Wochen

- Schenkelhalsfraktur: frühfunktionelle Mobilisierung mit Vollbelastung (bei Prothesenimplantation oder DHS/DHK) bzw. Teilbelastung bis $20 \mathrm{~kg}$ (bei Schraubenosteosynthese), bei erhöhtem Luxationsrisiko nach Prothesenimplantation Begrenzung der Flexion im Hüftgelenk auf $60^{\circ}$, keine Adduktion über die Mittellinie und Vermeidung forcierter (Innen-) Rotationsbewegungen im Hüftgelenk für die ersten sechs Wochen

- peri-/subtrochantäre Fraktur: frühfunktionelle Mobilisierung mit Vollbelastung ohne Limitierung der Hüftgelenkbeweglichkeit

- Thromboseausschluss mit Duplexsonografie oder Phlebografie 4-6 Tage postoperativ

- Entfernung des Hautverschlussmaterials am 14. postoperativen Tag

- radiologische Verlaufskontrollen nach sechs Wochen

- Einbindung des Sozialdienstes (Anschlussheilbehandlung/Kurzzeitpflege/häusliche Versorgung, Versorgung mit Hilfsmitteln wie Toilettensitzerhöhung, Keilkissen, Greifzange)

- bei jungem Patienten ggf. Implantatentfernung von Schrauben und extramedullären/zephalomedullären Implantaten nach sicher nachgewiesener (CT-Kontrolle) Konsolidierung der Fraktur

\section{Interessenkonflikt}

Die Autoren geben an, dass keine Interessenkonflikte vorliegen.
Autorinnen/Autoren

\section{Michael J. Raschke}

Prof. Dr. med., Klinik und Poliklinik für Unfall-, Hand- und Wiederhestellungschirurgie, Universitätsklinikum Münster

\section{Jens Everding}

Dr. med., Klinik und Poliklinik für Unfall-, Hand- und Wiederhestellungschirurgie, Universitätsklinikum Münster

\section{Korrespondenzadresse}

\section{Prof. Dr. Michael J. Raschke}

Klinik und Poliklinik für Unfall-, Hand- und Wiederhestellungschirurgie, Universitätsklinikum Münster

Albert-Schweitzer-Campus 1, Gebäude W1 48149 Münster

Michael.Raschke@ukmuenster.de

\section{Erstveröffentlichung}

Dieser Beitrag wurde erstveröffentlicht in: Günther KP, Hoffmann R, Hrsg. SOPs in der Orthopädie und Unfallchirurgie. Stuttgart: Thieme; 2017.

\section{Literatur}

[1] Bonnaire F, Lein T, Bula P. Trochanteric femoral fractures: anatomy, biomechanics and choice of implants. Unfallchirurg 2011; 114: 491-500

[2] Bonnaire FA, Weber AT. The influence of haemarthrosis on the development of femoral head necrosis following intracapsular femoral neck fractures. Injury 2002; 33 Suppl 3: C33-C40

[3] Droll KP, Broekhuyse H, O'Brien P. Fracture of the femoral head. J Am Acad Orthop Surg 2007; 15: 716-727

[4] Frerichmann U, Raschke M], Stockle U et al. Proximal femoral fractures in the elderly. Data from health insurance providers on more than 23 million insured persons-part 2. Unfallchirurg 2007; 110: 610-616

[5] Icks A, Haastert B, Wildner M et al. Trend of hip fracture incidence in Germany 1995-2004: a population-based study. Osteoporos Int 2008; 19: 1139-1145

[6] Liem IS, Kammerlander C, Raas C et al. Is there a difference in timing and cause of death after fractures in the elderly? Clin Orthop Relat Res 2013; 471: 2846-2851

[7] Lohmann R, Frerichmann U, Stockle U et al. Proximal femoral fractures in the elderly. Analysis of data from health insurance providers on more than 23 million insured persons - part 1 . Unfallchirurg 2007; 110: 603-609

[8] Papakostidis C, Panagiotopoulos A, Piccioli A et al. Timing of internal fixation of femoral neck fractures. A systematic review and meta-analysis of the final outcome. Injury 2015; 46: 459466

[9] Parker M], Dynan Y. Is Pauwels classification still valid? Injury 1998; 29: 521-523

[10] Parker M], Handoll HH. Gamma and other cephalocondylic intramedullary nails versus extramedullary implants for extracapsular hip fractures in adults. Cochrane Database Syst Rev 2010; (9): CD000093 
[11] Petersen MB, Jorgensen HL, Hansen K et al. Factors affecting postoperative mortality of patients with displaced femoral neck fracture. Injury 2006; 37: 705-711

[12] Smektala R, Grams A, Pientka L et al. Guidelines or state civil codes in the management of femoral neck fracture? An analysis of the reality of care provision in North Rhine-Westphalia. Dtsch Arztebl Int 2008; 105: 295-302

[13] Smektala R, Klaus H, Paech S. Trochanteric femur fractures analysis of external quality assurance within a comprehensive survey. Z Arztl Fortbild Qualitatssich 2005; 99: 537-545

[14] Stannard JP, Harris HW, Volgas DA et al. Functional outcome of patients with femoral head fractures associated with hip dislocations. Clin Orthop Relat Res 2000; 377: 44-56
[15] Stöckle U, Lucke M, Haas NP. Zertifizierte medizinische Fortbildung: Der Oberschenkelhalsbruch. Dtsch Arztebl 2005; 102: A-3426/B-2894/C-2710

[16] Taha ME, Audige L, Siegel G et al. Factors predicting secondary displacement after non-operative treatment of undisplaced femoral neck fractures. Arch Orthop Trauma Surg 2015; 135: 243-249

Bibliografie

DOI https://doi.org/10.1055/a-0602-6125

Orthopädie und Unfallchirurgie up2date 2019; 14: 120-126 (c) Georg Thieme Verlag KG Stuttgart · New York ISSN 1611-7859 